\title{
BMJ Open Fibrosis-4 index as a predictor for mortality in hospitalised patients with COVID-19: a retrospective multicentre cohort study
}

Jung Gil Park (D) , ${ }^{1}$ Min Kyu Kang (1) , ${ }^{1}$ Yu Rim Lee (1) , ${ }^{2}$ Jeong Eun Song, ${ }^{3}$ Na Young Kim, ${ }^{4}$ Young Oh Kweon, ${ }^{2}$ Won Young Tak, ${ }^{2}$ Se Young Jang (1) , ${ }^{2}$ Changhyeong Lee, ${ }^{3}$ Byung Seok Kim, ${ }^{3}$ Jae Seok Hwang, ${ }^{4}$ Byoung Kuk Jang, ${ }^{4}$ Jinmok Bae, ${ }^{4} \mathrm{Ji}$ Yeon Lee, ${ }^{4}$ Jeong III Suh, ${ }^{5}$ Soo Young Park (1) , ${ }^{2}$ Woo Jin Chung, ${ }^{4}$ On behalf of Daegu-Gyeongbuk Liver Study Group (DGLSG)

To cite: Park JG, Kang MK, Lee YR, et al. Fibrosis-4 index as a predictor for mortality in hospitalised patients with COVID-19: a retrospective multicentre cohort study. BMJ Open 2020;10:e041989. doi:10.1136/ bmjopen-2020-041989

- Prepublication history and supplemental material for this paper is available online. To view these files, please visit the journal online (http://dx.doi. org/10.1136/bmjopen-2020041989).

JGP and MKK are joint first authors.

Received 23 June 2020 Revised 01 September 2020 Accepted 25 September 2020

Check for updates

(c) Author(s) (or their employer(s)) 2020. Re-use permitted under CC BY-NC. No commercial re-use. See rights and permissions. Published by BMJ.

For numbered affiliations see end of article.

Correspondence to Professor Soo Young Park; psyoung0419@gmail.com and Professor Woo Jin Chung; chung50@dsmc.or.kr

\section{ABSTRACT}

Objective The reliable risk factors for mortality of COVID-19 has not evaluated in well-characterised cohort. This study aimed to identify risk factors for in-hospital mortality within 56 days in patients with severe infection of COVID-19.

Design Retrospective multicentre cohort study.

Setting Five tertiary hospitals of Daegu, South Korea.

Participants 1005 participants over 19 years old confirmed COVID-19 using real-time PCR from nasopharyngeal and oropharyngeal swabs.

Methods The clinical and laboratory features of patients with COVID-19 receiving respiratory support were analysed to ascertain the risk factors for mortality using the Cox proportional hazards regression model. The relationship between overall survival and risk factors was analysed using the Kaplan-Meier method.

Outcome In-hospital mortality for any reason within 56 days.

Results Of the 1005 patients, $289(28.8 \%)$ received respiratory support, and of these, 70 patients $(24.2 \%)$ died. In multivariate analysis, high fibrosis-4 index (FIB-4; HR 2.784), low lymphocyte count (HR 0.480), diabetes (HR 1.917) and systemic inflammatory response syndrome (HR 1.714) were found to be independent risk factors for mortality in patients with COVID-19 receiving respiratory support (all $p<0.05$ ). Regardless of respiratory support, survival in the high FIB-4 group was significantly lower than in the low FIB-4 group ( 28.8 days vs 44.0 days, respectively, $p<0.001)$. A number of risk factors were also significantly related to survival in patients with COVID-19 regardless of respiratory support ( $0-4$ risk factors, 50.2 days; 49.7 days; 44.4 days; 32.0 days; 25.0 days, respectively, $p<0.001$ ).

Conclusion FIB-4 index is a useful predictive marker for mortality in patients with COVID-19 regardless of its severity.

\section{INTRODUCTION}

COVID-19, caused by SARS-CoV-2, is one of the most important healthcare concerns

\section{Strengths and limitations of this study}

Use of simple scoring system widely used in clinical practice.

- Predict mortality regardless of its severity.

- Very low probability of sampling bias.

- Requiring further studies for validation with other cohorts.

- Relatively early cohort before outbreak caused by newer variant of COVID-19.

worldwide. ${ }^{1}$ It was first identified in Wuhan City, Hubei province, central China, and linked to Wuhan's Huanan Seafood Wholesale Market in December 2019. ${ }^{2}$ To investigate the causative pathogen, pan-CoV PCR was performed initially, followed by metagenomics analysis using next-generation sequencing. ${ }^{3}$ After commercial real-time quantitative PCR-based detection methods became available, the number of patients with confirmed COVID-19 rapidly increased. ${ }^{4}$ Subsequently, this outbreak spread internationally and was recognised as a pandemic by the WHO on 11 March $2020 .^{5}$

In South Korea, 3705 patients were diagnosed in the Daegu and Gyeongsangbuk-do area among a total of 4212 patients diagnosed in South Korea from 19 January to 2 March 2020. ${ }^{6}$ Epidemiological surveillance revealed that $2333(63.0 \%)$ of cases in this area were related to the religious group Shincheonji. ${ }^{6}$ At that time, the mortality rate was only $0.5 \%$ in South Korea and $0.6 \%$ in the Daegu and Gyeongsangbuk-do area. ${ }^{7}$ However, by 28 April, the mortality rate had increased to 224 of 10752 confirmed cases $(2.3 \%){ }^{8}$

As a result of unprecedented demand, most countries are experiencing a shortage 
of medical resources. The difficulty of dealing with this emergency would be assisted by earlier diagnosis, as well as forecasts of mortality. Several Chinese studies of clinical characteristics and risk factors relating to COVID-19 have been published, ${ }^{49-13}$ and recently, the clinical and epidemiological experience of several other countries have been reported. ${ }^{1415}$ However, few reports of risk modelling and prediction are awaiting peer review and publication, ${ }^{16}$ and most of these studies have limited sample size or high risk of bias. ${ }^{16}$ The risk prediction models in these studies were established using conventional scoring systems, risk nomograms or advanced machine learning models. ${ }^{17-19}$ Although the performance of such models is relatively good, no COVID-19 risk prediction model can currently be recommended for clinical use due to a number of limitations. ${ }^{20}$

This study aims to evaluate the predictive risk factors for mortality by analysing epidemiological and laboratory features in patients with COVID-19 receiving respiratory support in tertiary hospitals within the Daegu and Gyeongsangbuk-do area. Most cases in South Korea were concentrated in this area, the most severe cases being admitted to five tertiary hospitals.

\section{MATERIALS AND METHODS}

\section{Patients and their public involvement}

After the beginning of the COVID-19 outbreak on 18 February 2020, in Daegu, all patients with COVID-19 were admitted to one designated tertiary hospital. Because of limited medical resources, efficient allocation was required. Therefore, from 2 March, the disinfection team of Daegu classified all new patients with COVID-19 on the basis of severity of respiratory symptoms and oxygen demand to transfer to one of four other tertiary hospitals in accordance with regional policy. Accordingly, from 20 February to 14 April 2020, we enrolled 1005 hospitalised patients aged $>19$ years with COVID-19 confirmed by PCR in five tertiary hospitals of Daegu, South Korea.

Written informed consent by the patients was waived due to the retrospective nature of our study. It was not possible to involve patients or the public in the design, conduct, reporting or dissemination plans of this study.

\section{Data collection}

The medical records of anthropometric and epidemiological data, patients' clinical characteristics, radiological and laboratory data, treatments, use of ACE inhibitors or angiotensin II receptor blockers (ARBs) and clinical outcomes were collected retrospectively by each hospital and reviewed by two independent reviewers. Laboratory tests included complete blood cell and lymphocyte count, erythrocyte sedimentation rate (ESR), $\mathrm{C}$ reactive protein (CRP), liver and kidney function tests, electrolytes and serum ferritin on admission day. All patients underwent chest radiography with or without CT. Antivirals, hydroxychloroquine, systemic glucocorticoid, intravenous immunoglobulin, respiratory support, continuous renal replacement therapy and extracorporeal membrane oxygenation (ECMO) were included on the treatment of COVID-19. The data collection terminated on 14 April 2020.

\section{Definition}

In accordance with the WHO interim guidance, all COVID-19 cases were diagnosed by detection of SARS-CoV-2 sequence using real-time PCR from nasopharyngeal and oropharyngeal swabs. Fever was defined as tympanic temperature of $37.5^{\circ} \mathrm{C}$ or higher. Systemic inflammatory response syndrome (SIRS) on admission was defined by satisfaction of any two of the criteria: (A) white cell count (WCC) $<4000$ cells $/ \mathrm{mm}^{3}$ or $>12000$ cells / $\mathrm{mm}$, (B) body temperature $<36^{\circ} \mathrm{C}$ or $>38^{\circ} \mathrm{C}$, (C) heart rate $>90$ beats $/ \mathrm{min}$ and (D) tachypnoea $>20$ breaths/ min. Persistent hypotension was defined by MAP $<65 \mathrm{~mm}$ $\mathrm{Hg}$ despite volume resuscitation, requiring vasopressors to maintain MAP. Acute respiratory distress syndrome (ARDS) were defined in accordance with the WHO interim guidance. Acute kidney injury was defined either from the highest serum creatinine level $(>0.3 \mathrm{mg} / \mathrm{dL}$ within 48 hours or 1.5 times of the baseline level within 7 days $)$ and/or from decreased urine output $(<0.6 \mathrm{~mL} /$ $\mathrm{kg} /$ hour for 6 hours) on admission. In accordance with oxygen demand, two groups of respiratory support were defined as low-dose oxygen group using nasal cannula or venturi mask and high-dose oxygen group using highflow nasal cannula, invasive mechanical ventilation and/ or ECMO.

Chronic liver disease was defined by chronic hepatitis $\mathrm{B}$ or $\mathrm{C}$ infection, liver cirrhosis and hepatocellular carcinoma by history taking or serology test. The fibrosis-4 index (FIB-4), which is calculated from age, aspartate aminotransferase (AST), alanine aminotransferase (ALT) and platelet counts, was originally used to predict liver fibrosis in patients with chronic liver disease. FIB-4 was assessed as: age $($ year $) \times$ AST $(\mathrm{U} / \mathrm{L}) /$ [platelet count $\left.\left(10^{9} / \mathrm{L}\right) \times \sqrt{ } \operatorname{ALT}(\mathrm{U} / \mathrm{L})\right]^{21}$

\section{Study outcomes}

The primary objective of this study was to identify predictive risk factors for in-hospital mortality for any reason within 56 days in patients with COVID-19 receiving respiratory support. The secondary objective was to evaluate whether FIB-4 index is associated with mortality in patients with COVID-19 regardless of respiratory support.

\section{Statistical analysis}

All continuous data were expressed as mean and SD (mean $\pm \mathrm{SD}$ ) or median with range, and compared using Student's t-test or the Mann-Whitney U test. Categorical data were compared using a $\chi^{2}$ test or Fisher's exact test. The predictive factors for mortality were assessed using the Cox proportional hazards regression model with HR using backward selection method. Receiver operating characteristic (ROC) analysis was conducted to assess the predictive performance of assessed risk factors. The 


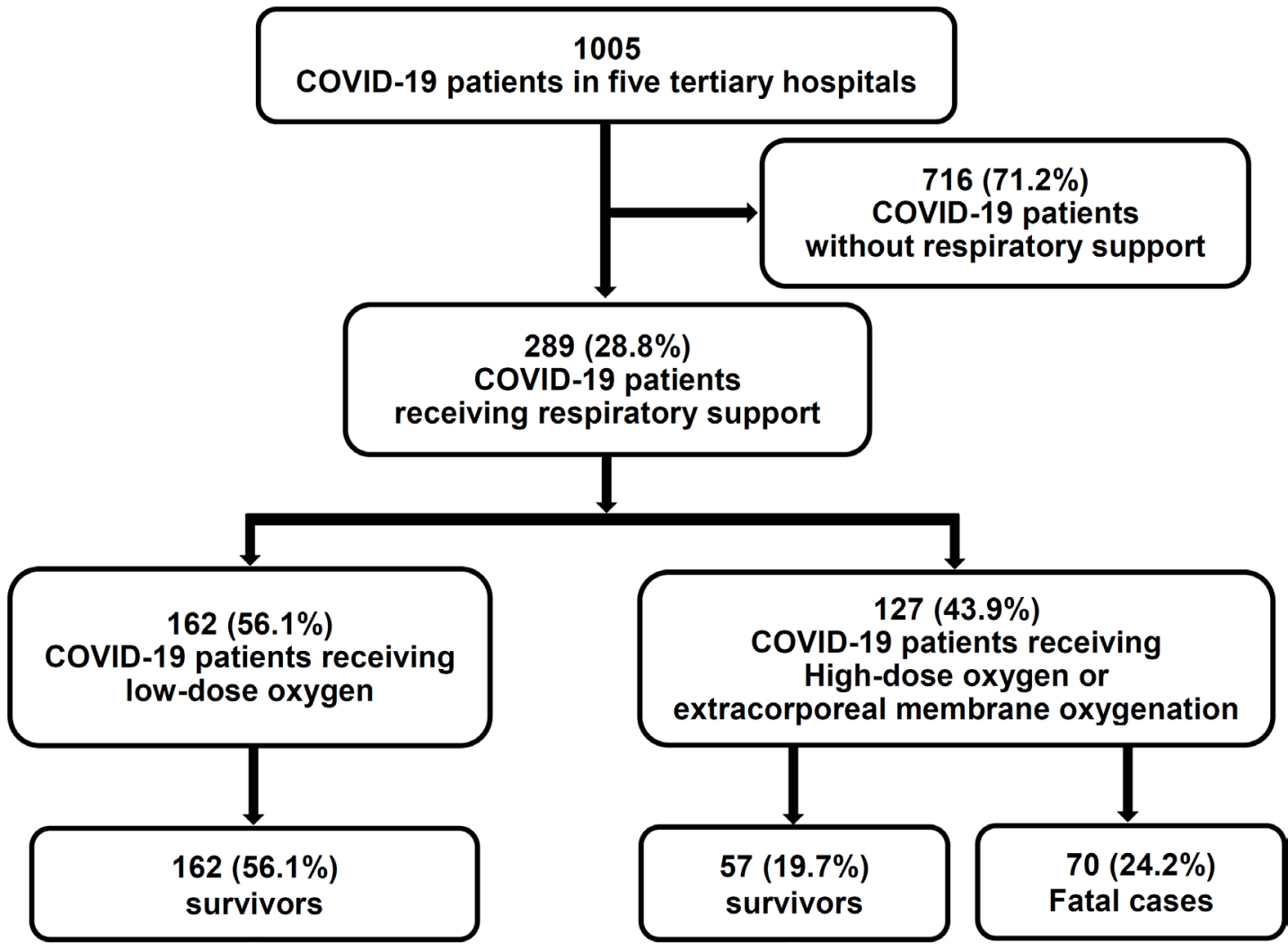

Figure 1 Flow diagram of the study.

best cut-off values (COVs) were calculated based on the Youden index. The relationship between overall survival and FIB-4 was calculated using the Kaplan-Meier method. The valuables including age, AST, ALT and platelet, which were used for calculation of FIB-4, were not included in the multivariate analysis to avoid multicollinearity. $\mathrm{P}$ value $<0.05$ was considered statistically significant. All statistical analyses were performed using $\mathrm{R}$ (version 3.0, http://cran.r-project.org/, install. packages(“devtools")) software.

\section{RESULTS}

\section{Baseline characteristics}

Of 1005 patients with COVID-19, 289 (28.8\%) received respiratory support and were included in this study. Of these, 162 $(56.1 \%)$ were treated with low-dose oxygen therapy using a nasal or venturi mask, while $127(43.9 \%)$ were treated with a high-flow nasal mask, invasive mechanical ventilation and/or ECMO. Patient disposition is shown in figure 1. The baseline characteristics of the patients with COVID-19 receiving respiratory support are shown in table 1 . There were several differences in demographics and history between fatal cases and survivors, including older age, preponderance of males and more frequent diabetes among fatal cases. However, there was no significant difference in the use of ACE inhibitors or ARBs. Duration of symptoms before admission was shorter in survivors, but the presence of fever or respiratory symptoms on admission did not differ between survivors and nonsurvivors. There was no significant difference in viral signs on admission except for frequent SIRS in fatal cases. Differences in some laboratory tests on admission were significant, including higher white cell count, CRP, procalcitonin, AST, gamma glutamyl transferase, prothrombin time, blood urea nitrogen, serum creatinine, and lower lymphocyte count, platelet count, serum albumin, and serum sodium in fatal cases compared with survivors.

\section{Treatments and clinical outcomes}

The treatments and clinical outcomes of patients with COVID-19 receiving respiratory support are shown in table 2. Of these, $57(19.7 \%)$ and $70(24.2 \%)$ were treated with high-flow nasal cannula and invasive mechanical ventilation, respectively. Analysis of clinical outcomes revealed that 113 patients (39.1\%) had ARDS, 93 (33.2\%) were admitted to an intensive care unit (ICU) and 18 (6.2\%) underwent ECMO. The median duration of hospital stay was 25 days (range 8-33). Survivors were less frequently treated with darunavir/cobicistat, systemic glucocorticoid, high-flow nasal cannula, invasive mechanical ventilation or continuous renal replacement therapy compared with fatal cases. Survivors had a longer median duration of hospital stay compared with fatal cases, were less frequently admitted to an ICU and less frequently developed persistent hypotension, ARDS or acute kidney injury.

Risk factors for mortality in patients with COVID-19 receiving respiratory support

Univariate analysis identified age, sex, diabetes, chronic obstructive pulmonary disease, lymphocyte count, AST, 
Table 1 Baseline characteristics of patients with COVID-19 receiving respiratory support

$\begin{array}{llll}\left.\begin{array}{l}\text { All } \\ (n=289\end{array} \text { to } 100 \%\right) & \begin{array}{l}\text { Survivors } \\ (n=219 \text { to } 75.8 \%)\end{array} & \begin{array}{l}\text { Fatal cases } \\ (n=70 \text { to } 24.2 \%)\end{array} & \text { P value* }\end{array}$

Demographic and clinical characteristics

\begin{tabular}{|c|c|c|c|c|}
\hline Age, years & $72.0(62.0-80.0)$ & $70.0(60.0-79.0)$ & $77.0(71.0-84.0)$ & $<0.001$ \\
\hline Female gender & $156(54.0)$ & $128(58.4)$ & $28(40.0)$ & 0.011 \\
\hline Body mass index, $\mathrm{kg} / \mathrm{m}^{2}$ & $24.3(22.2-26.3)$ & $24.2(22.2-26.2)$ & $24.5(22.2-26.8)$ & 0.577 \\
\hline \multicolumn{5}{|l|}{ Comorbidity } \\
\hline Hypertension & $132(45.8)$ & $96(44.0)$ & $36(51.4)$ & 0.346 \\
\hline Diabetes & 93 (32.3) & $59(27.1)$ & $34(48.6)$ & 0.001 \\
\hline Cardiovascular disease & $20(6.9)$ & $14(6.4)$ & $6(8.6)$ & 0.723 \\
\hline $\begin{array}{l}\text { Chronic obstructive pulmonary } \\
\text { disease }\end{array}$ & $9(3.1)$ & $4(1.8)$ & $5(7.1)$ & 0.067 \\
\hline Chronic kidney disease & $11(3.8)$ & $9(4.1)$ & $2(2.9)$ & 0.906 \\
\hline Chronic liver disease & $15(5.2)$ & $9(4.1)$ & $6(8.6)$ & 0.248 \\
\hline Liver cirrhosis & $8(2.8)$ & $4(1.8)$ & $4(5.7)$ & 0.191 \\
\hline Hepatocellular carcinoma & $2(0.7)$ & $1(0.5)$ & $1(1.4)$ & 0.979 \\
\hline ACE inhibitors or ARBs use & $61(24.8)$ & $47(24.1)$ & $14(27.5)$ & 0.756 \\
\hline \multicolumn{5}{|l|}{ Symptoms on admission } \\
\hline Fever/chills & $195(67.9)$ & $145(66.5)$ & $50(72.5)$ & 0.438 \\
\hline Cough & $175(61.2)$ & $139(63.8)$ & $36(52.9)$ & 0.145 \\
\hline Shortness of breath & $152(53.0)$ & $112(51.4)$ & $40(58.0)$ & 0.413 \\
\hline $\begin{array}{l}\text { Gastrointestinal symptoms } \\
\text { (vomiting/diarrhoea) }\end{array}$ & $73(25.4)$ & $67(30.7)$ & $6(8.7)$ & $<0.001$ \\
\hline Myalgia & $88(30.8)$ & $73(33.5)$ & $15(22.1)$ & 0.103 \\
\hline Headache & $46(16.1)$ & $43(19.7)$ & $3(4.4)$ & 0.005 \\
\hline $\begin{array}{l}\text { Duration of symptom before } \\
\text { admission, days }\end{array}$ & $6(3-9)$ & $6(3-9)$ & $4.5(2-7)$ & 0.031 \\
\hline \multicolumn{5}{|l|}{ Vital signs at presentation } \\
\hline Temperature, ${ }^{\circ} \mathrm{C}$ & $36.9(36.5-37.6)$ & $37.0(36.5-37.6)$ & $36.7(36.5-37.3)$ & 0.070 \\
\hline $\begin{array}{l}\text { Respiratory rate, } \\
\text { breath/min }\end{array}$ & $20(20-22)$ & $20(20-22)$ & $20(20-23)$ & 0.101 \\
\hline Saturation, \% & 95 (92-98) & 95 (93-98) & $95(90-100)$ & 0.948 \\
\hline Systolic pressure, $\mathrm{mm} \mathrm{Hg}$ & $130(116-145)$ & $130(120-144)$ & $122(108-146)$ & 0.062 \\
\hline Heart rate, /min & $86(72-100)$ & $85(72-97)$ & $92(72-102)$ & 0.140 \\
\hline SIRS on admission & $102(35.7)$ & $65(30.1)$ & $37(52.9)$ & 0.001 \\
\hline \multicolumn{5}{|l|}{ Radiological and laboratory findings } \\
\hline \multicolumn{5}{|l|}{ Radiological findings } \\
\hline Abnormal chest radiograph & $269(93.1)$ & $201(91.8)$ & $68(97.1))$ & 0.205 \\
\hline $\begin{array}{l}\text { Bilateral involvement } \\
\text { on chest radiographs }\end{array}$ & $225(83.6)$ & $163(81.1)$ & $62(91.2)$ & 0.080 \\
\hline \multicolumn{5}{|l|}{ Laboratory findings } \\
\hline White cell count, $\times 10^{3} / \mu \mathrm{L}$ & $6140(4695-8065)$ & $6000(4690-7420)$ & $7320(5100-12020)$ & 0.001 \\
\hline Lymphocyte count, $\times 10^{3} / \mu \mathrm{L}$ & $895(611-1260)$ & $952(661-1321)$ & $702(490-980)$ & $<0.001$ \\
\hline Haemoglobin, g/dL & $12.4(11.1-13.6)$ & $12.4(11.2-13.6)$ & $12.6(10.9-13.9)$ & 0.510 \\
\hline Platelet count, $\times 10^{9} / \mathrm{L}$ & $192(146-267)$ & $200(150-277)$ & 166 (132-239) & 0.029 \\
\hline $\begin{array}{l}\text { Erythrocyte sedimentation rate, } \\
\mathrm{mm} / \text { hour }\end{array}$ & $57(39-76)$ & $57(39-76)$ & $51(40-70)$ & 0.592 \\
\hline C reactive protein, $\mathrm{mg} / \mathrm{L}$ & $10.1(4.8-21.5)$ & $9.3(4.0-20.4)$ & $13.4(7.4-24.8)$ & 0.015 \\
\hline
\end{tabular}


Table 1 Continued

\begin{tabular}{|c|c|c|c|c|}
\hline & $\begin{array}{l}\text { All } \\
(n=289 \text { to } 100 \%)\end{array}$ & $\begin{array}{l}\text { Survivors } \\
(\mathrm{n}=219 \text { to } 75.8 \%)\end{array}$ & $\begin{array}{l}\text { Fatal cases } \\
(n=70 \text { to } 24.2 \%)\end{array}$ & P value* \\
\hline Procalcitonin, ng/mL & $0.1(0.1-0.4)$ & $0.1(0.1-0.2)$ & $0.4(0.1-1.1)$ & $<0.001$ \\
\hline Alanine aminotransferase, U/L & $21(15-33)$ & $20(15-32)$ & $23(16-38)$ & 0.528 \\
\hline Total bilirubin, mg/dL & $0.6(0.4-0.9)$ & $0.6(0.4-0.9)$ & $0.7(0.4-0.9)$ & 0.240 \\
\hline $\begin{array}{l}\text { Gamma glutamyl transferase, } \\
\text { U/L }\end{array}$ & $35(22-61)$ & $27(16.5-48.5)$ & $60(40-101)$ & 0.001 \\
\hline Serum albumin, $\mathrm{g} / \mathrm{dL}$ & $3.4(3.2-3.7)$ & $3.5(3.2-3.8)$ & $3.2(3.0-3.4)$ & $<0.001$ \\
\hline Prothrombin time, $s$ & $12.4(11.8-13.3)$ & $12.4(11.7-13.1)$ & $12.8(11.9-14.8)$ & 0.026 \\
\hline Prothrombin time, INR & $1.1(1.0-1.1)$ & $1.0(1.0-1.1)$ & $1.1(1.0-1.3)$ & 0.015 \\
\hline Sodium, mmol/L & $137(134-141)$ & $138(134-141)$ & $136(133-140)$ & 0.006 \\
\hline Potassium, mmol/L & $4.1(3.7-4.5)$ & $4.1(3.7-4.5)$ & $4.2(3.5-4.7)$ & 0.870 \\
\hline Lactate dehydrogenase, U/L & $558(405-753)$ & $560(404-753)$ & $556(410-762)$ & 0.969 \\
\hline Creatine kinase, U/L & $79(52-155)$ & $73(51-149)$ & $86(54-172)$ & 0.307 \\
\hline Serum ferritin, $\mathrm{ng} / \mathrm{mL}$ & $552(327-975)$ & $430(308-941)$ & 659 (521-1432) & 0.115 \\
\hline
\end{tabular}

Data are expressed as median and IQR or numbers (\%).

${ }^{*}$ Calculated by Student's t-test (or the Mann-Whitney $U$ test, if appropriate) and $\chi^{2}$ test (or Fisher's exact test, if appropriate).

ARB, angiotensin II receptor blocker; SIRS, systemic inflammatory response syndrome.

ESR and SIRS as significant variables relating to mortality in patients with COVID-19 receiving respiratory support (table 3). Multivariate analysis identified age (HR 1.054; 95\% CI 1.028 to $1.082 ; \mathrm{p}<0.001$ ), diabetes (HR 2.226; $95 \%$ CI 1.357 to $3.652 ; \mathrm{p}=0.002$ ), low lymphocyte count (HR 0.999; $95 \%$ CI 0.998 to $1.000 ; \mathrm{p}=0.005$ ) and high AST (HR 1.002; 95\% CI 1.000 to 1.003 ; $\mathrm{p}=0.033$ ) as independent predictors of mortality. Lower platelet count (HR $0.997 ; 95 \%$ CI 0.994 to $1.000, \mathrm{p}=0.069)$ and presence of SIRS on admission (HR 1.968; 95\% CI 1.199 to 3.230; $\mathrm{p}=0.074$ ) tended to be associated with severe COVID-19, but this did not reach statistical significance.

\section{Risk factors for mortality, including FIB-4 index}

Based on multivariate analysis, we used FIB-4 as a predictive risk factor candidate. In multivariate analysis including FIB-4 as a continuous variable, diabetes (HR 1.998 ; $95 \%$ CI 1.202 to $3.321 ; \mathrm{p}=0.008$ ), lower lymphocyte count (HR $0.999 ; 95 \%$ CI 0.998 to $1.000 ; \mathrm{p}=0.003$ ) and FIB-4 (HR 1.115; 95\% CI 1.069 to 1.163 ; $\mathrm{p}<0.001$ ) were identified as independent predictors of mortality in patients with COVID-19 receiving respiratory support (online supplemental table S1). To set a COV of FIB-4 and lymphocyte count, ROC analysis was performed (figure 2). The areas under the ROC curves of FIB-4 and lymphocyte counts were 0.702 and 0.647 with sensitivity of $48.5 \%$ and $78.6 \%$, specificity of $87.6 \%$ and $45.8 \%$, positive predictive value of $55.0 \%$ and $32.0 \%$ and negative predictive value of $84.4 \%$ and $86.9 \%$, respectively (all $\mathrm{p}<0.001$ ). The optimal COVs of FIB-4 and lymphocyte count were 4.95 and 1010 , respectively.

In multivariate analysis after converting FIB-4 and lymphocyte count to categorical variables, diabetes (HR 1.917; $95 \%$ CI 1.181 to 3.111; $\mathrm{p}=0.009$ ), low lymphocyte count (HR 0.480; 95\% CI 0.271 to $0.852 ; \mathrm{p}=0.012$ ), SIRS (HR $1.714 ; 95 \%$ CI 1.048 to 2.802; $\mathrm{p}=0.032$ ) and high FIB-4 (HR 2.784; 95\% CI 1.691 to 4.585 ; $\mathrm{p}<0.001$ ) were identified as independent predictors of mortality (table 4). In addition, the results of high FIB-4 as a predictor of survival was consistent in stepwise multivariate analysis (online supplemental table S2).

\section{FIB-4 and other predictive risk factors for survival in patients} with COVID-19 receiving respiratory support

Among the four predictive risk factors, FIB- 4 was the best predictor of mortality in patients with COVID-19 receiving respiratory support. Therefore, we performed survival analysis to compare mortality in the high FIB-4 group (FIB-4 $\geq 4.95$ ) and low FIB-4 group (FIB-4 <4.95). Survival in the high FIB-4 group was significantly lower than in the low FIB-4 group (high FIB-4: 28.8 days (23.8-33.8); low FIB-4: 44.0 days (41.9-46.1), $\mathrm{p}<0.001$ ) (figure 3A.) Using the four variables diabetes, lymphocyte count, SIRS on admission and FIB-4, we performed survival analysis to predict mortality in patients 
Table 2 Treatments and clinical outcomes of patients with COVID-19 receiving respiratory support

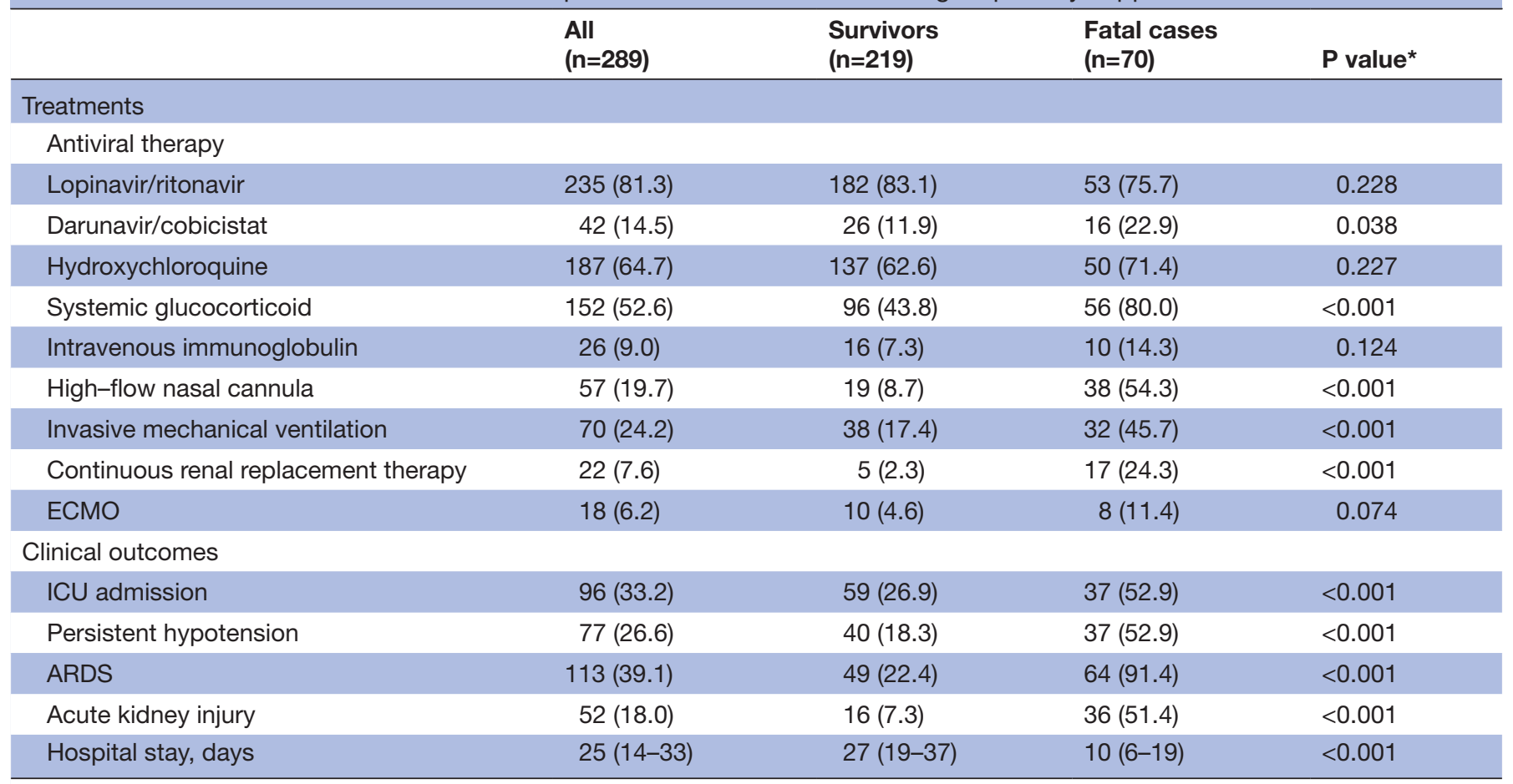

Data are expressed as median and IQR or numbers (\%).

${ }^{*}$ Calculated by Student's t test (or the Mann-Whitney U test, if appropriate) and $\chi^{2}$ test (or Fisher's exact test, if appropriate).

ARDS, acute respiratory distress syndrome; ECMO, extracorporeal membrane oxygenation; ICU, intensive care unit.

with COVID-19 receiving respiratory support. As the number of risk factors increased, survival of the patients significantly deteriorated (no risk factor: 47.3 days (44.2-50.4); one risk factor: 40.7 days (37.4-44.0); two risk factors: 38.0 days (33.8-42.2); three risk factors, 30.7 days (23.9-37.5); four risk factors, 25.0 days $(6.3-43.7), \mathrm{p}=0.0016$ (figure $3 \mathrm{~B}$, online supplemental figure S1.

To explore additional predictive performance for mortality in the entire group of patients with COVID-19, we performed survival analysis to compare mortality in the high and low FIB-4 groups using the same cut-off. Survival in the high FIB-4 group was significantly lower than in the low FIB-4 group (high FIB-4: 32.5 days (27.7-37.2) and low FIB-4: 50.0 days (49.3-50.6), $\mathrm{p}<0.001$ ) (figure 4A.) Using four variables, we also performed survival analysis to predict mortality in the entire group of patients with COVID-19. As the number of risk factors increased, survival significantly deteriorated (no risk factor: 50.2 days (48.6-51.70; one risk factor: 49.7 days (48.8-50.5); two risk factors; 44.4 days (942.2-46.6); three risk factors: 32.0 days (25.7-38.3); four risk factors: 25.0 days (6.3-43.7), $\mathrm{p}<0.001$ (figure 4B) (online supplemental figure S2). In patients with COVID-19 receiving high-dose oxygen, survival in the high FIB-4 group was significantly lower than in the low FIB-4 group (high FIB-4: 16.5 days (7.0-32.0); low FIB-4: 20.0 days (10.0-33.0), $\mathrm{p}=0.011$ ) (data not shown).

\section{DISCUSSION}

In this retrospective cohort study, predictive risk factors for mortality were evaluated in 289 patients with confirmed
COVID-19 receiving respiratory support in the Daegu and Gyeongsangbuk-do area. Diabetes, low lymphocyte count, SIRS and FIB-4 were revealed as independent risk factors for mortality in COVID-19. Furthermore, survival of patients with low FIB-4 and number of risk factors is better than those with high FIB-4 and number of risk factors. A recent meta-analysis found that the main laboratory abnormalities in patients with COVID-19 included low lymphocyte count and elevated CRP and lactate dehydrogenase $(\mathrm{LDH}){ }^{22}$ In non-survivors, or severely ill patients requiring ICU care or suffering from ARDS, laboratory abnormalities including high WCC count, low lymphocyte count, prolonged prothrombin time, low albumin, elevated AST, ALT, total bilirubin, $\mathrm{LDH}$, creatinine, troponin I, CRP, procalcitonin, ferritin and D-dimer were identified as risk factors in previous studies. ${ }^{9}{ }^{1012}$ However, numbers of enrolled patients were small, and multivariate analyses were not performed. In a recent study, logistic regression analysis identified age, Sequential Organ Failure Assessment (SOFA) score and D-dimer as predictive risk factors for death in patients with COVID-19 pneumonia. ${ }^{13}$ SOFA score is derived from arterial oxygen tension (or pressure)/fractional inspired oxygen, use of mechanical ventilator, platelets count, Glasgow Coma scale, bilirubin, mean arterial pressure or requirement for vasoactive agents and serum creatinine or urine output. The score is related to the cytokine storm in sepsis, ${ }^{23}$ and we think some of the risk factors in our study, including platelets as a component 
Table 3 Risk factors for mortality in patients with COVID-19 receiving respiratory support

\begin{tabular}{|c|c|c|c|}
\hline \multirow[b]{2}{*}{ Variable } & \multirow{2}{*}{$\begin{array}{l}\text { Univariate } \\
\text { P value* }\end{array}$} & \multicolumn{2}{|c|}{ Multivariate analysis } \\
\hline & & P value* & HR (95\% Cl) \\
\hline Age, years & $<0.001$ & $<0.001$ & 1.054 (1.028 to 1.082$)$ \\
\hline \multicolumn{4}{|l|}{ Comorbidities (yes/no) } \\
\hline Hypertension & 0.392 & & \\
\hline Chronic obstructive pulmonary disease & 0.009 & & \\
\hline Chronic kidney disease & 0.841 & & \\
\hline Chronic liver disease & 0.226 & & \\
\hline ACE inhibitor/ARB use (yes/no) & 0.871 & & \\
\hline Lymphocyte count, $\times 10^{3} / \mathrm{uL}$ & $<0.001$ & 0.005 & $0.999(0.998$ to 1.000$)$ \\
\hline Alanine aminotransferase, $U / L$ & 0.552 & & \\
\hline Total bilirubin, mg/dL & 0.831 & & \\
\hline Alkaline phosphatase, U/L & 0.725 & & \\
\hline Gamma glutamyl transferase, U/L & 0.263 & & \\
\hline Serum albumin, g/dL & 0.773 & & \\
\hline Prothrombin time, INR & 0.444 & & \\
\hline Estimated glomerular filtration rate, $\mathrm{mL} / \mathrm{min} / 1.73 \mathrm{~m}^{2}$ & 0.002 & & \\
\hline SIRS on admission (yes/no) & $<0.001$ & 0.074 & 1.968 (1.199 to 3.230$)$ \\
\hline
\end{tabular}

${ }^{*}$ Calculated by Cox proportional hazards regression test.

ARB, angiotensin II receptor blocker; INR, international normalised ratio; SIRS, systemic inflammatory response syndrome.

of the FIB-4 index, and SIRS, were also associated with this serious inflammatory condition. This recent study included patients similar to those in the present study, as judged from the proportion of patients receiving respiratory support $(82.1 \%$ vs $100 \%$ in the present study) and the mortality rate ( $28.3 \%$ vs $24.2 \%$ in the present study). ${ }^{13}$

A.

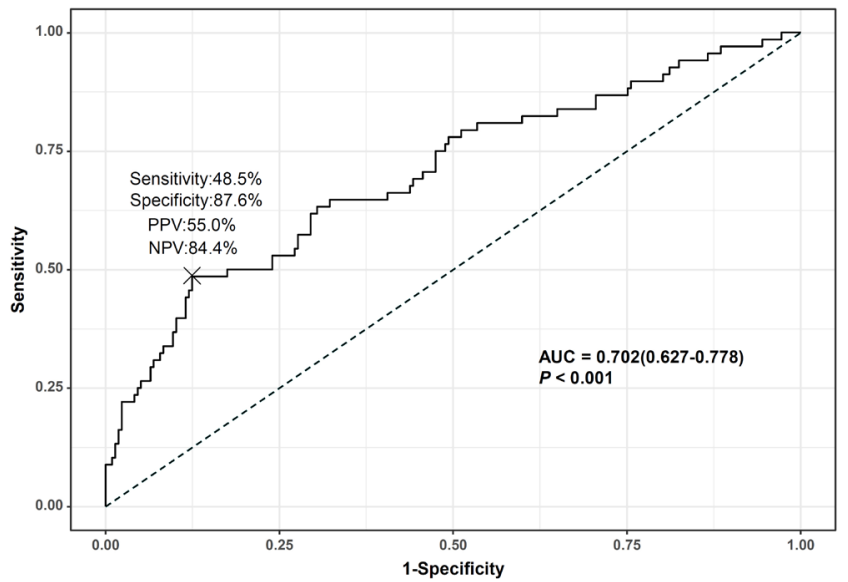

Multivariate analysis in two recent studies showed that neutrophil-to-lymphocyte ratio, $\mathrm{CD} 4 \mathrm{~T}$ cell count and age were independent risk factors of in-hospital mortality and ICU admission for COVID-19. ${ }^{24}{ }^{25}$ Severe inflammation dysregulates the immune response and is characterised by decreased memory helper $\mathrm{T}(\mathrm{Th})$ cells and regulatory $\mathrm{T}$

B.

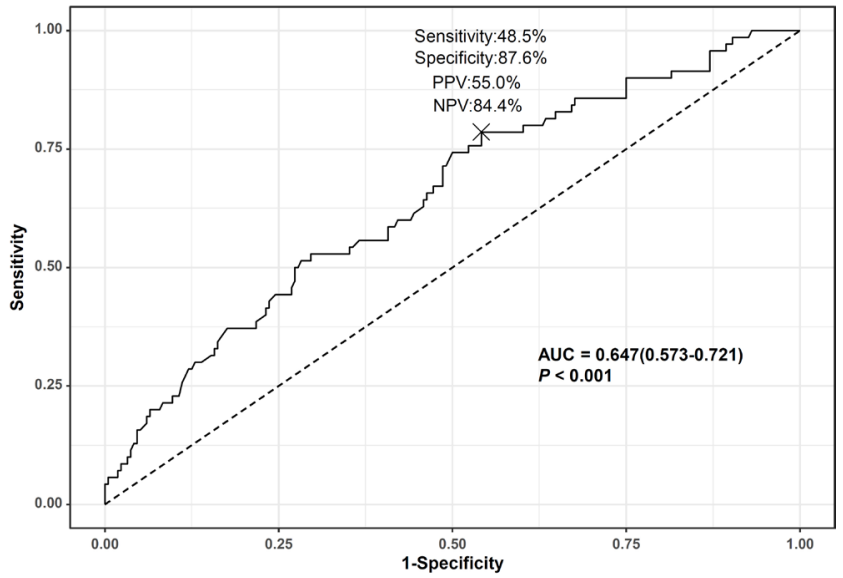

Figure 2 Predictive performance of risk factors for mortality in patients with COVID-19 receiving respiratory support. (A) Area under the curve (AUC) for fibrosis-4 index; (B) area under the curve for lymphocyte counts. NPV, negative predictive value; PPV, positive predictive value. 
Table 4 Risk factors including fibrosis-4 index for mortality in patients with COVID-19 receiving respiratory support

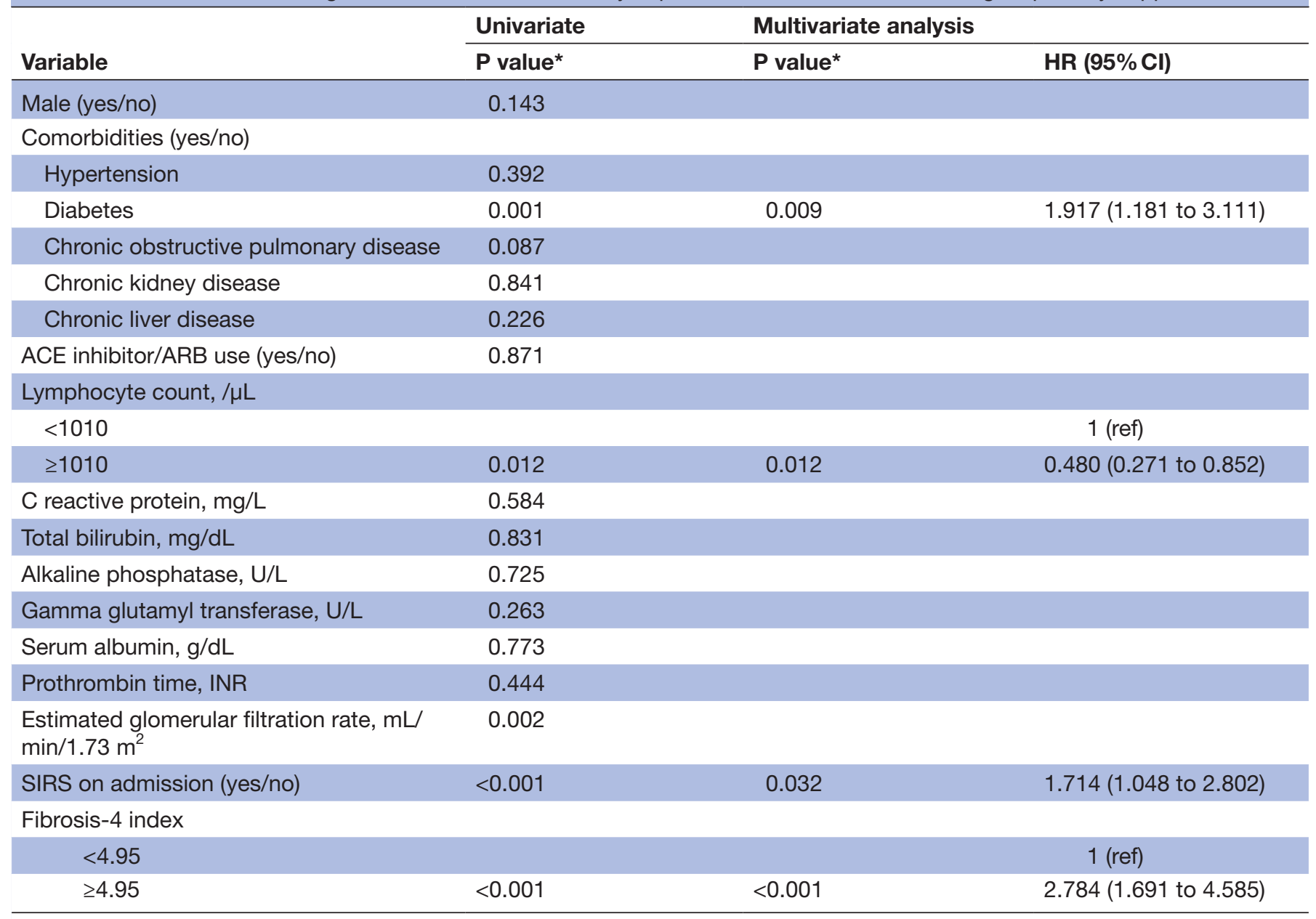

${ }^{*}$ Calculated by Cox proportional hazards regression test.

ARB, angiotensin II receptor blocker; INR, international normalised ratio; SIRS, systemic inflammatory response syndrome.

cells with increased naive Th cells in patients with COVID$19 .^{26}$ These findings are consistent with low lymphocyte count as an independent risk factor for mortality in our study. However, in previous studies, survival analysis was not performed, and the enrolled patients were somewhat different from those in the present study.

Liver injury in COVID-19 was observed more frequently in severe cases than in mild cases. ${ }^{4} 12$ Though the

A

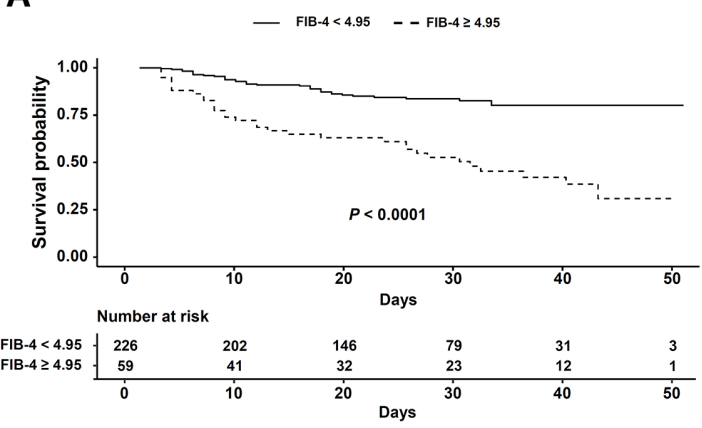

mechanism is unclear, elevated AST and ALT may be related to the immune response in severe pneumonia, which may result from inflammatory cytokines following COVID-19 infection. ${ }^{27}$ Elevated liver enzyme can be also associated with drug-induced liver injury (DILI), which may result from antibacterial and antiviral drugs, antiinflammatory drugs and vasopressors in severe cases. ${ }^{28}$ As there has been no study of DILI in COVID-19 infection,
B - No risk factor --1 risk factor --2 risk factors ---3 risk factors $\cdots 4$ risk factors

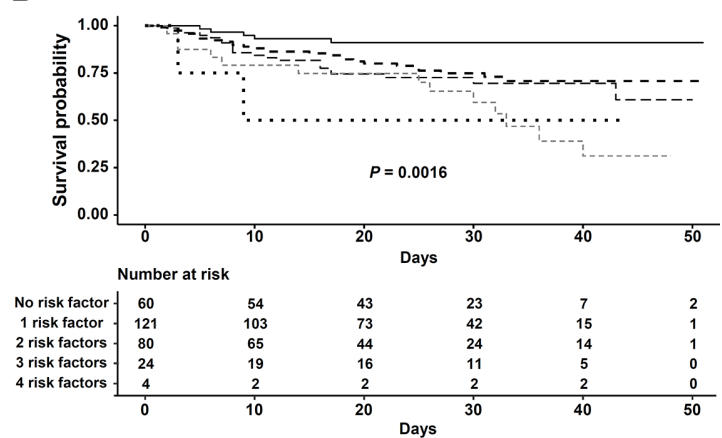

Figure 3 Survival of patients with COVID-19 receiving respiratory support plotted against fibrosis-4 (FIB-4) index (A) and number of risk factors (B). 
A

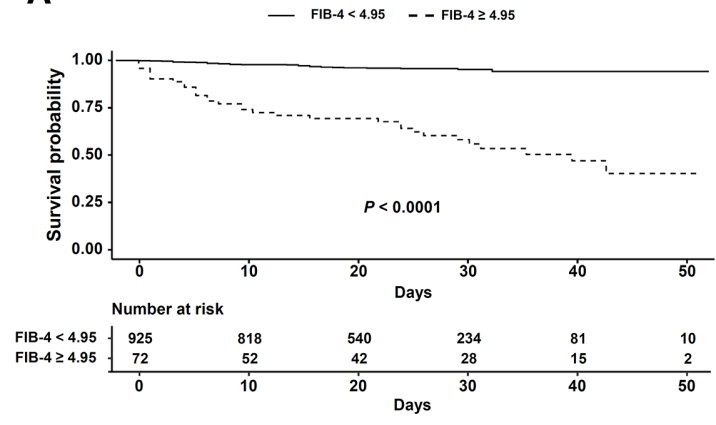

B
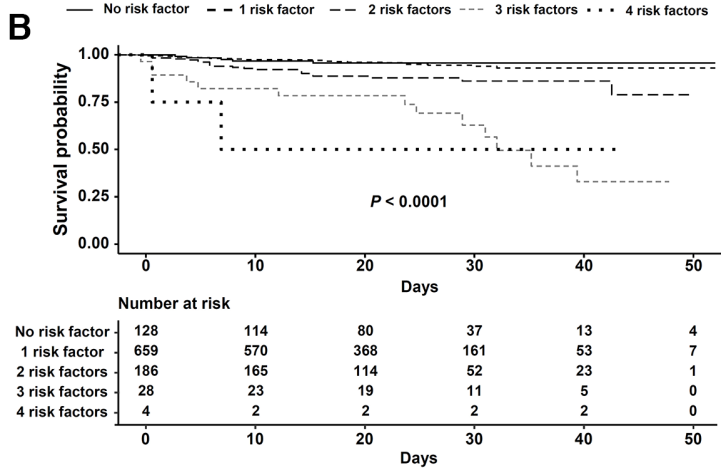

Figure 4 Survival of patients with COVID-19 plotted against fibrosis-4 (FIB-4) index (A) and number of risk factors (B).

its prevalence should be investigated. However, in this study, laboratory tests performed at the time of admission did not indicate an association between AST elevation and DILI. Also in this study, although FIB-4 was originally used in patients with liver disease, it was identified as a predictor of mortality in patients with COVID-19, whether they were receiving respiratory support. Elevated $\mathrm{LDH}$ has been reported as a promising predictor for severe COVID-19 infection. ${ }^{18}{ }^{29}$ However, it was only identified as a risk factor by univariate analysis, not by multivariate analysis. We suggest that the ratio of AST to ALT in FIB-4 may be a better predictor of mortality than the level of LDH, due to its non-specificity of cause. In addition, the common finding of elevated AST in patients with severe disease in several other studies supports the present study. ${ }^{42}{ }^{31}$ Recently, association of FIB-4 with ICU admission in patients with COVID-19 was reported in Spain. ${ }^{32}$ They calculated FIB-4 using laboratory tests at the same time of SARS-CoV-2 detection to assess presence of advanced fibrosis. However, although they exclude previously diagnosed patients with myopathies and platelets disorders to avoid non-specificity of FIB-4, values of AST, ALT and platelets can be affected by COVID-19 infection itself. Furthermore, as described above, severe cause of COVID-19 infection can affect AST and platelet more than mild case. If they overcome these, we think they should use laboratory test to estimate advanced fibrosis before patients had COVID-19.

When FIB-4 is analysed with other risk factors including lymphocyte count, SIRS, and diabetes, as number of risk factors increases, survival deteriorates in patients with COVID-19 regardless of respiratory support. There are several published or preprinted studies of prediction models for the prognosis of patients with COVID-19. ${ }^{16}$ Albumin, direct bilirubin and red blood cell distribution width have been suggested as diagnostic or prognostic indicators of severe disease or mortality in COVID-19. ${ }^{16}$ However, among these three factors, albumin was not a significant risk factor, and the other two factors were not evaluated in the present study. Most of the proposed models have been open to criticism on the grounds of severe sampling bias due to rarely reported length of follow-up and prevalence of COVID-19 with or without severe infection. A strength of the present study is the low probability of sampling bias, because approximately three-fourths of patients with COVID-19 in South Korea have been diagnosed in the Daegu and Gyeongsangbuk-do area, and our entire cohort was derived from tertiary hospitals in that area.

This study has some limitations. First, there was no validation with another cohort. As described above, most of the COVID-19 cases were enrolled in this study. Thus, it would be impossible to validate these results without undertaking an international study. Improved assessments of international data on COVID-19 will require data sharing, using a reporting protocol specified by WHO. ${ }^{33}$ Second, detailed radiological assessment of CT scans was not performed. To our knowledge, there are only a few reports at preprint stage that include clinical features and radiological features from CT scan with artificial intelligence techniques to develop prediction models. ${ }^{34}$ However, this study also has sampling bias as well as an inadequate sample size. ${ }^{20}{ }^{34}$ Therefore, advanced machine learning combining radiological image analysis with clinical risk factors would be needed to develop a robust prediction model. Third, prediction of severe COVID-19 including ICU admission or ARDS was not analysed in this study. However, we think prediction of severe COVID-19 was not appropriate for our cohort, because transfer to a tertiary hospital may introduce the possibility of sampling bias. Thus, we used the objective outcome of mortality in this study.

In conclusion, FIB-4, diabetes, low lymphocyte count and SIRS are independent risk factors of mortality in patients with COVID-19 receiving respiratory support. Among these risk factors, FIB-4 is a robust predictor of survival in patients with COVID-19 regardless of respiratory support. A number of risk factors are significantly related to survival in patients with COVID-19 regardless of respiratory support.

\section{Author affiliations}

${ }^{1}$ Department of Internal Medicine, College of Medicine, Yeungnam University, Daegu, South Korea

${ }^{2}$ Department of Internal Medicine, School of Medicine, Kyungpook National University, Kyungpook National University Hospital, Daegu, South Korea ${ }^{3}$ Department of Internal Medicine, School of Medicine, Daegu Catholic University, Daegu, South Korea

${ }^{4}$ Department of Internal Medicine, School of Medicine, Keimyung University, Daegu, South Korea

${ }^{5}$ Department of Internal Medicine, College of Medicine, Dongguk University, Dongguk University Gyeongju Hospital, Gyeongju, South Korea 
Acknowledgements We appriciate all dedicated doctors and epidemiologist who contribute declining trends of patients with COVID-19 in the Daegu and Gyeongsangbuk-do area and Editage (www.editage.co.kr) for English language editing.

Contributors Conceptualisation: SYP and WJC; formal analysis: MKK; data curation: MKK, YRL, JES and NYK; investigation: JGP, MKK, YRL, JES, NYK, YOK, WYT, SYJ, CL, BSK, JSH, BKJ,JB, JYL, JIS and SYP; supervision: SYP and WJC; visualisation: JGP; writing - original draft: JGP and MKK; writing - review and editing: SYP and WJC.

Funding This work was supported by the 2020 Yeungnam University Research Grant.

\section{Competing interests None declared.}

Patient consent for publication Not required.

Ethics approval This study was performed in accordance with the ethical guidelines of the revised Helsinki Declaration of 2013 and approved by the Institutional Review Board of all tertiary hospitals.

Provenance and peer review Not commissioned; externally peer reviewed.

Data availability statement Data are available on reasonable request. The data that support the findings of this study are also available from the corresponding author (SYP and WJC) on reasonable request.

Supplemental material This content has been supplied by the author(s). It has not been vetted by BMJ Publishing Group Limited (BMJ) and may not have been peer-reviewed. Any opinions or recommendations discussed are solely those of the author(s) and are not endorsed by BMJ. BMJ disclaims all liability and responsibility arising from any reliance placed on the content. Where the content includes any translated material, BMJ does not warrant the accuracy and reliability of the translations (including but not limited to local regulations, clinical guidelines, terminology, drug names and drug dosages), and is not responsible for any error and/or omissions arising from translation and adaptation or otherwise.

Open access This is an open access article distributed in accordance with the Creative Commons Attribution Non Commercial (CC BY-NC 4.0) license, which permits others to distribute, remix, adapt, build upon this work non-commercially, and license their derivative works on different terms, provided the original work is properly cited, appropriate credit is given, any changes made indicated, and the use is non-commercial. See: http://creativecommons.org/licenses/by-nc/4.0/.

\section{ORCID iDs}

Jung Gil Park http://orcid.org/0000-0001-5472-4731

Min Kyu Kang http://orcid.org/0000-0002-1435-3312

Yu Rim Lee http://orcid.org/0000-0003-1916-1448

Se Young Jang http://orcid.org/0000-0001-9148-9670

Soo Young Park http://orcid.org/0000-0002-4944-4396

\section{REFERENCES}

1 Mason RJ. Pathogenesis of COVID-19 from a cell biology perspective. Eur Respir J 2020;55:13993003.00607-2020:2000607.

$2 \mathrm{Lu} \mathrm{H}$, Stratton CW, Tang Y-W. Outbreak of pneumonia of unknown etiology in Wuhan, China: the mystery and the miracle. J Med Virol 2020:92:401-2.

3 Zhou P, Yang X-L, Wang X-G, et al. A pneumonia outbreak associated with a new coronavirus of probable bat origin. Nature 2020;579:270-3.

4 Guan WJ, ZY N, Hu Y, et al. Clinical characteristics of coronavirus disease 2019 in China. N Engl J Med2020.

5 World Health Organization. WHO Director-General's opening remarks at the media briefing on COVID-19-11 March 2020". Available: https://www.who.int/dg/speeches/detail/who-director-general-sopening-remarks-at-the-media-briefing-on-covid-19-11-march-2020 [Accessed 11 Mar 2020].

6 Korean Society of Infectious Diseases, Korean Society of Pediatric Infectious Diseases, Korean Society of Epidemiology, et al. Report on the epidemiological features of coronavirus disease 2019 (COVID-19) outbreak in the Republic of Korea from January 19 to March 2, 2020. $J$ Korean Med Sci 2020;35:e112.

7 Korean Society of Infectious Diseases and Korea Centers for Disease Control and Prevention. Analysis on 54 mortality cases of coronavirus disease 2019 in the Republic of Korea from January 19 to March 10, 2020. J Korean Med Sci 2020;35:e132.

8 Korea Centers for Disease Control and Prevention. The update of COVID-19 in Korea as of April 28. Available: https://www.cdc.go.kr/ board/board.es?mid=a30402000000\&bid=https://www.cdc.go.kr/ board/board.es? $\mathrm{mid}=\mathrm{a} 30402000000 \& \mathrm{bid}=00300030$

9 Wu C, Chen X, Cai Y, et al. Risk factors associated with acute respiratory distress syndrome and death in patients with coronavirus disease 2019 pneumonia in Wuhan, China. JAMA Intern Med 2020.

10 Wang D, Hu B, Hu C, et al. Clinical characteristics of 138 hospitalized patients with 2019 novel coronavirus-infected pneumonia in Wuhan, China. JAMA 2020;323:1061-9.

11 Chen N, Zhou M, Dong X, et al. Epidemiological and clinical characteristics of 99 cases of 2019 novel coronavirus pneumonia in Wuhan, China: a descriptive study. Lancet 2020;395:507-13.

12 Huang C, Wang Y, Li X, et al. Clinical features of patients infected with 2019 novel coronavirus in Wuhan, China. Lancet 2020;395:497-506.

13 Zhou F, Yu T, Du R, et al. Clinical course and risk factors for mortality of adult inpatients with COVID-19 in Wuhan, China: a retrospective cohort study. Lancet 2020;395:1054-62.

14 Grasselli G, Zangrillo A, Zanella A, et al. Baseline characteristics and outcomes of 1591 patients infected with SARS-CoV-2 admitted to ICUs of the Lombardy region, Italy. JAMA 2020;323:1574-81.

15 Gudbjartsson DF, Helgason A, Jonsson H, et al. Spread of SARSCoV-2 in the Icelandic population. N Engl J Med 2020;382:2302-15.

16 Wynants L, Van Calster B, Collins GS, et al. Prediction models for diagnosis and prognosis of covid-19 infection: systematic review and critical appraisal. BMJ 2020;369:m1328.

17 Shi Y, Yu X, Zhao H, et al. Host susceptibility to severe COVID-19 and establishment of a host risk score: findings of 487 cases outside Wuhan. Crit Care 2020;24:108.

18 Gong J, Ou J, Quu X, et al. A Tool to Early Predict Severe Corona Virus Disease 2019 (COVID-19) : A Multicenter Study using the Risk Nomogram in Wuhan and Guangdong, China. Clin Infect Dis 2020.

19 Caramelo F, Ferreira N, Oliveiros B. Estimation of risk factors for COVID-19 mortality - preliminary results. medRxiv 2020.

20 Sperrin M, Grant SW, Peek N. Prediction models for diagnosis and prognosis in Covid-19. BMJ 2020;369:m1464.

21 Sterling RK, Lissen E, Clumeck N, et al. Development of a simple noninvasive index to predict significant fibrosis in patients with HIV/ HCV coinfection. Hepatology 2006;43:1317-25.

22 Fu L, Wang B, Yuan T, et al. Clinical characteristics of coronavirus disease 2019 (COVID-19) in China: a systematic review and metaanalysis. J Infect 2020.

23 Marik PE, Taeb AM. Sirs, qSOFA and new sepsis definition. J Thorac Dis 2017;9:943-5.

24 Liu Y, Du X, Chen J, et al. Neutrophil-To-Lymphocyte ratio as an independent risk factor for mortality in hospitalized patients with COVID-19. J Infect2020.

25 Chen J, Qi T, Liu L, et al. Clinical progression of patients with COVID-19 in Shanghai, China. J Infect 2020;80:e1-6.

26 Qin C, Zhou L, Hu Z, et al. Dysregulation of immune response in patients with coronavirus 2019 (COVID-19) in Wuhan, China. Clin Infect Dis 2020;71:762-8.

27 Fan Z, Chen L, Li J, et al. Clinical features of COVID-19-Related liver damage. J Gastroenterol Hepatol 2020.

28 Qi X, Liu C, Jiang Z, et al. Multicenter analysis of clinical characteristics and outcome of COVID-19 patients with liver injury. $J$ Hepatol 2020.

29 Yan L, Zhang H-T, Xiao Y, et al. Prediction of survival for severe Covid-19 patients with three clinical features: development of a machine learning-based prognostic model with clinical data in Wuhan. medRxiv 2020.

30 Xie J, Hungerford D, Chen $\mathrm{H}$, et al. Development and external validation of a prognostic multivariable model on admission for hospitalized patients with COVID-19. medRxiv 2020.

31 Shi H, Han X, Jiang N, et al. Radiological findings from 81 patients with COVID-19 pneumonia in Wuhan, China: a descriptive study. Lancet Infect Dis 2020;20:425-34.

32 Ibáñez-Samaniego L, Bighelli F, Usón C, et al. Elevation of liver fibrosis index FIB-4 is associated with poor clinical outcomes in patients with COVID-19. J Infect Dis 2020;222:726-33.

33 Moorthy V, Henao Restrepo AM, Preziosi M-P, et al. Data sharing for novel coronavirus (COVID-19). Bull World Health Organ 2020;98:150.

34 Bai X, Fang C, Zhou Y, et al. Predicting COVID-19 malignant progression with Al techniques. SSRN Journal 2020. 